\title{
AMENDMENTS
}

\section{Publisher Correction: Real-space observation of vibrational strong coupling between propagating phonon polaritons and organic molecules}

Andrei Bylinkin (D), Martin Schnell (D), Marta Autore, Francesco Calavalle, Peining Li, Javier Taboada-Gutièrrez (D), Song Liu, James H. Edgar (iD, Fèlix Casanova (D), Luis E. Hueso (iD), Pablo Alonso-Gonzalez (D), Alexey Y. Nikitin (D) and Rainer Hillenbrand (D)

Correction to: Nature Photonics https://doi.org/10.1038/s41566-020-00725-3, published online 23 November 2020.

In the version of this Letter originally published, the $x$-axis label of Fig. $3 \mathrm{f}$ incorrectly read ' $L_{\mathrm{Pp}} / v_{\mathrm{gr}} \kappa$ (ps)'; it should have read ' $L_{\mathrm{PP}} / v_{\mathrm{gr}} \tau(\mathrm{ps})$ '. This has now been corrected in all versions of the Letter.

Published online: 4 December 2020

https://doi.org/10.1038/s41566-020-00746-y

๑ The Author(s), under exclusive licence to Springer Nature Limited 2020 